Meta

Journal des traducteurs

Translators' Journal

\title{
Porte ouverte sur la documentation scientifique et technique en langue française
}

\section{Michel Saint-Laurent}

Volume 25, numéro 1, mars 1980

La documentation

URI : https://id.erudit.org/iderudit/004295ar

DOI : https://doi.org/10.7202/004295ar

Aller au sommaire du numéro

Éditeur(s)

Les Presses de l'Université de Montréal

ISSN

0026-0452 (imprimé)

1492-1421 (numérique)

Découvrir la revue

Citer cet article

Saint-Laurent, M. (1980). Porte ouverte sur la documentation scientifique et technique en langue française. Meta, 25(1), 174-177.

https://doi.org/10.7202/004295ar d'utilisation que vous pouvez consulter en ligne.

https://apropos.erudit.org/fr/usagers/politique-dutilisation/ 


\title{
Porte ouverte sur la documentation scientifique et technique en langue française
}

\author{
Michel SAint-LAuRENT \\ agent d'information, \\ Informatech France-Québec
}

Informatech France-Québec a pour mission de diffuser l'information scientifique et technique de langue française. La situation nord-américaine du Québec permet une diffusion abondante de la documentation en langue anglaise. Il n'en demeure pas moins que la documentation en langue française répond à plusieurs besoins ainsi qu'on peut en juger par la diversité des utilisateurs d'Informatech France-Québec. Les traducteurs sont de ceux-là. Plusieurs d'entre eux profitent des services d'Informatech pour compléter leur documentation de base sur les sujets scientifiques et techniques qu'ils ont à traiter.

Environ 19 entreprises engagées dans le domaine de la traduction sont inscrites comme clients d'Informatech. Ce chiffre ne comprend pas les organismes gouvernementaux ou autres qui ont des services de traduction et qui utilisent les services d'Informatech, à des fins de traduction en plus de les utiliser pour la recherche proprement dite.

Informatech France-Québec est né en 1966 d'ententes entre la France et le Québec dans les domaines technique et scientifique. En 1974, les accords entre les premiers ministres Bourassa et Chirac définissaient l'organisme comme le maître d'œuvre de l'information scientifique et technique de langue française au Québec.

La documentation, tant française que québécoise, est disponible par l'entremise d'Informatech. Les deux pays peuvent donc profiter d'un éventail élargi de documentation en langue française. On doit noter qu'on trouve aussi de nombreuses références à des documents rédigés en d'autres langues dont bien sûr l'anglais. Ce qui est exclusivement en français, ce ne sont pas les documents contenus dans les divers fonds documentaires, mais bien les références leur donnant accès.

\section{Un centre automatisé de documentation}

Pour traiter la documentation qui venait au centre, Informatech dut concevoir un logiciel-maison : SABINE (Système informatisé de banques d'information non numériques). SABINE dialogue avec ses utilisateurs en mode conversationnel. C'est-à-dire qu'il utilise des mots clés français et que toutes ses opérationsréponses sont données en français. À cause de cette caractéristique, on le confond parfois avec BADADUQ. La fonction de même que le contenu de chacun sont bien différents. BADADUQ ne porte que sur le fonds documentaire des biblio- 
thèques de l'Université du Québec. SABINE est le seul système au Québec à offrir en français des banques spécialisées.

Une banque d'information documentaire est un ensemble de références à des documents qui peuvent être situés dans divers centres de documentation. Pour un sujet donné, lutilisateur obtiendra de SABINE, le titre du document, le nom de son auteur, toutes les références bibliographiques habituelles (maison et lieu d'édition, année, volume, pages et/ou chapitre, mention de traduction) et un résumé analytique du document.

\section{Accès direct}

Toute cette information est accessible aux entreprises équipées d'un terminal, soit par liaison téléphonique, soit par l'intermédiaire du réseau DATAPAC. Cette dernière option réduit les coûts pour l'utilisateur éloigné de Montréal. La plupart des grandes entreprises possèdent l'équipement et le personnel qualifié pour utiliser un logiciel tel que SABINE. L'une des grandes qualités de SABINE est sa simplicité, laquelle ne l'empêche pas d'effectuer le même travail dans le domaine de l'information documentaire que les grands ordinateurs. Informatech offre aux utilisateurs de l'accès direct une session de formation et un manuel-guide qui permettra un dialogue efficace avec SABINE.

\section{Les banques disponibles}

Chaque banque est baptisée d'un nom qui, déjà, donne une idée des sujets dont elle traite. AGRIDOQ donne accès à plus de 50000 références du domaine de l'agro-alimentaire; TEXTILDOQ concerne le textile (30000 références); URBADOQ constitue le seul fichier de langue française au Canada sur l'urbanisme, l'environnement et l'aménagement (11 000 références) ; FRANCIS porte sur les sciences humaines (135000 références) ; enfin PASCAL, la plus grosse des banques, compte 500000 références.

En ce qui concerne FRANCIS, les domaines traités sont les sciences de l'éducation, la sociologie et l'ethnologie, les sciences du langage, les sciences administratives. PASCAL (Programme appliqué à la sélection et à la compilation automatique de la littérature) est un réseau multidisciplinaire réunissant 15 banques ; il a été mis à la disposition du Québec grâce à la coopération d'Informatech France-Québec et du CNRS (Centre national de la recherche scientifique) en France.

SABINE offre aussi RADAR (Répertoire analytique des articles de revues du Québec), la première banque québécoise à faire partie du centre de documentation. Bientôt ENVIRODOQ, sur l'environnement, viendra ajouter au contenu québécois.

\section{Les bulletins signalétiques}

Le service de documentation reçoit chaque mois 500 revues techniques de langue française qu'il dépouille et dont certains articles forment la base des 12 bulletins signalétiques spécialisés qui paraissent chaque mois. Ils se veulent très 
DONNEES STATISTIQUES SUR LES BASES OFFERTES

\begin{tabular}{|c|c|c|c|}
\hline Banques & Sujets & $\begin{array}{l}\text { Nombre de } \\
\text { références } \\
\text { Fin } 1978\end{array}$ & $\begin{array}{l}\text { Volume et } \\
\text { fréquence des } \\
\text { mises à jour }\end{array}$ \\
\hline PASCAL 1 & $\begin{array}{l}\text { Biologie et Physiologie végétales - Sylvi- } \\
\text { culture - Protozoaires et Invertébrés - Zoo- } \\
\text { logie générale et appliquée }\end{array}$ & 32400 & $\begin{array}{l}2200 \text { réf. } \\
\text { Mens. }\end{array}$ \\
\hline PASCAL 2 & $\begin{array}{l}\text { Minéralogie - Géochimie - Géologie extra- } \\
\text { terrestre - Gisements métalliques et non } \\
\text { métalliques - Économie minière } \\
\text { Hydrologie - Géologie de l'ingénieur et for- } \\
\text { mations superficielles }\end{array}$ & 103500 & $\begin{array}{l}1600 \text { réf. } \\
\text { Mens. }\end{array}$ \\
\hline PASCAL 3 & $\begin{array}{l}\text { Science de l'information - Documentation } \\
\text { Analyse numérique - Informatique - Auto- } \\
\text { matique - Recherche opérationnelle - Ges- } \\
\text { tion } \\
\text { Électrotechnique } \\
\text { Électronique }\end{array}$ & 228000 & $\begin{array}{l}3300 \text { réf. } \\
\text { Mens. }\end{array}$ \\
\hline PASCAL 6 & $\begin{array}{l}\text { Polymères - Peintures - Bois - Cuirs } \\
\text { Génie chimique - Industries chimique et } \\
\text { parachimique } \\
\text { Nuisances }\end{array}$ & 138600 & $\begin{array}{l}3000 \text { réf. } \\
\text { Mens. }\end{array}$ \\
\hline PASCAL 7 & $\begin{array}{l}\text { Combustibles - Énergie } \\
\text { Economie de l'énergie }\end{array}$ & 98000 & $\begin{array}{l}1600 \text { réf. } \\
\text { Mens. }\end{array}$ \\
\hline PASCAL 8 & $\begin{array}{l}\text { Métaux - Métallurgie } \\
\text { Soudage - Brasage et techniques connexes } \\
\text { Industries mécaniques }- \text { Bâtiment - Tra- } \\
\text { vaux publics - Transports }\end{array}$ & 217000 & $\begin{array}{l}3200 \text { réf. } \\
\text { Mens. }\end{array}$ \\
\hline PASCAL 9 & World Trans Index & 10000 & - \\
\hline AGRIDOQ & Agro-alimentaire & 52500 & $\begin{array}{l}1200 \text { réf. } \\
\text { Mens. }\end{array}$ \\
\hline URBADOQ & Urbanisme - Aménagement & 11000 & $\begin{array}{l}200 \text { réf. } \\
\text { Mens. }\end{array}$ \\
\hline TEXTILDOQ & Industrie textile & 30000 & - \\
\hline RADAR & Multidisciplinaire & 35000 & $\begin{array}{l}1300 \text { réf. } \\
\text { Bimens. }\end{array}$ \\
\hline FRANCIS & $\begin{array}{l}\text { Sciences de l'éducation } \\
\text { Sociologie - Ethnologie } \\
\text { Sciences du langage } \\
\text { Science administrative }\end{array}$ & $\begin{array}{l}47400 \\
45400 \\
24450 \\
17000\end{array}$ & $\begin{array}{l}750 \text { réf. } \\
8 / \text { an } \\
800 \text { réf. } \\
8 / \text { an } \\
480 \text { réf. } \\
8 / \text { an } \\
425 \text { réf. } \\
8 / \text { an }\end{array}$ \\
\hline
\end{tabular}


succincts, et les documents cités sont disponibles aux bureaux d'Informatech. Les bulletins traitent des domaines suivants : urbanisme, aménagement et environnement, agro-alimentaire, textiles, énergie - nuisances, transports - génie civil, sciences physiques, informatique - automatique, métallurgie - mécanique, gestion - économie, électronique - électrotechnique.

\section{Disponibilité des documents}

Généralement, le délai de livraison d'un article n'excède pas 48 heures. Par ailleurs, si Informatech ne possède pas tous les documents signalés par SABINE, un système efficace permet de les obtenir. Informatech participe aux prêts interbibliothèques et peut se charger de commander, reproduire et envoyer tout document, y compris ceux qui ne sont pas disponibles en Amérique du Nord. Dans le cas d'un article commandé auprès d'une bibliothèque ou d'un centre de documentation, le délai de livraison est fonction de l'éloignement géographique.

\section{S'affirmer comme instrument complémentaire}

La quantité phénoménale de documentation traitée chaque jour dans le monde rend indispensable le recours à l'automatisation. Celle-ci permet de répertorier une grande quantité de documents et en outre, elle aide l'utilisateur à faire un choix de documents restreint mais pertinent. $\AA$ une époque où l'informatique s'impose dans tous les domaines, elle peut aussi être un instrument de travail précieux pour le traducteur. Il n'en tient qu'à lui de s'y intéresser.

Parmi les géants américains de la documentation automatisée, Informatech France-Québec trouve sa place. Non seulement cet organisme rend accessible la documentation scientifique et technique française, mais il participe au développement de banques documentaires québécoises qu'il intégrera à son système de diffusion. En plus d'intégrer ENVIRODOQ à son fonds documentaire, Informatech collabore à la constitution d'HISCABEQ sur l'histoire du Canada et de BIBLIOCOM sur les sciences de la communication. 